\title{
TIPIFICAÇÃO DOS RESERVATÓRIOS DE USINAS HIDRELÉTRICAS EM MINAS GERAIS
}

Mariana d'Ávila Fonseca Paiva de Paula Freitas ${ }^{1}$

\begin{abstract}
Resumo
O presente artigo apresenta a tipificação dos reservatórios de usinas hidrelétricas do estado de Minas Gerais. A matriz energética do Brasil é em sua maioria proveniente da hidroeletricidade, portanto estudos nessa área são válidos para assegurar proteção do potencial hidráulico e do aproveitamento hidrelétrico. A tipificação consiste no agrupamento de ambientes em tipos com características geográficas e hidromorfológicas semelhantes para permitir a avaliação de condições ecológicas. Esse tipo de classificação surgiu na Europa, sendo o principal instrumento da Política da União Europeia relativo aos recursos hídricos. No estudo foram contempladas variáveis de 19 reservatórios no Estado. A formação dos grupos de reservatórios foi realizada por meio de procedimentos estatísticos que inicialmente reduziu a quantidade de variáveis (Análise de Componentes Principais) e a partir da seleção das variáveis mais representativas foi feita análise de cluster para o agrupamento de reservatórios. Este trabalho é parte de um projeto que posteriormente validará o processo de tipificação com resultados biológicos. A classificação, além do foco ecológico de avaliar as condições dos ambientes, também servirá de subsídio na gestão dos reservatórios em Minas Gerais.
\end{abstract}

Palavras-chave: reservatórios, tipificação, ambientes aquáticos.

\begin{abstract}
Thispaperpresentsthecharacterization of reservoirs of hydroelectric in Minas Gerais. Brazil'senergymatrixismostlycomingfromhydroelectricity, sostudies in thisarea are important for conferringprotection of hydraulic and hydroelectricpotential. The classificationisthegrouping of environmentstypeswith similar geographical and hydromorphologicalcharacteristicstoallowtheassessment of ecologicalconditions. Thistype of classificationstarted in Europe, and it isthemaininstrument of EUpolicyaboutwater. Thisstudy covers variables of 19 reservoirs of the State. The formation of reservoirsgroupswasperformedusingstatistical procedures. First, reducedtheamount of variables (Principal ComponentAnalysis) and fromtheselection of themostrepresentativevariableswasperformed cluster analysis for groupingreservoirs. Thisworkispart of a projectthatfurthervalidatestheprocess of typingwithbiologicalresults. The classification, beyondtheecologicalfocus of assessingtheconditions of theenvironment, will serve as a subsidy in the management of reservoirs in Minas Gerais.
\end{abstract}

Keywords:reservoir, typology, aquaticenvironments

1Universidade Federal de Minas Gerais - Instituto de Geociências (Discente)

Instituto SENAI de Tecnologia em Meio Ambiente - SENAI FIEMG

(marianadfreitas@gmail.com)

Cadernos do Leste

Artigos Cientificos

Belo Horizonte, Jan.-Dez. Vol.16, n¹6, 2016 


\section{1- INTRODUÇÃO}

O Brasil possui grande disponibilidade hídrica, que possibilita um alto aproveitamento hidrelétrico. Como consequência, a matriz energética do país é essencialmente composta por hidrelétricas, de acordo com a Agência Nacional de Energia Elétrica - Aneel (2015). A energia hidrelétrica é obtida por meio do aproveitamento do potencial hidráulico de um rio. Para isso é preciso construir usinas em cursos de água com desníveis do relevo e que tenham volume de água abundante.

Segundo Tundisi (2003), a construção de represas é uma das mais antigas e importantes intervenções humanas nos sistemas naturais. Uma das principais alterações em um corpo hídrico é a modificação do escoamento, que altera um ambiente de águas correntes (lótico) para de águas com fluxo reduzido ou parado. Os reservatórios podem ter uso como reserva de água para hidroeletricidade, navegação, irrigação, águas para abastecimento público e controle de cheias. De acordo com Campagnoli e Diniz (2012), no Brasil existem cerca de 420 reservatórios em operação.

As usinas hidrelétricas são classificadas de acordo com sua potência e área do reservatório (Aneel apud Energisa, 2015):

- $\mathrm{CHH}$ - Centrais Geradoras Hidrelétricas: até $1 \mathrm{MW}$ de potência instalada, a fio d'água e normalmente com barragem somente para desvio do curso de água;

- PCH - Pequenas Centrais Hidrelétricas: entre 1,1 MW e 30 MW de potência instaladas e reservatórios com área de no máximo $3 \mathrm{~km}^{2}$;

- UHE - Usina Hidrelétrica de Energia: mais de 30 MW de potência instalada e reservatório com mais de $3 \mathrm{~km}^{2}$.

Como o intuito deste trabalho é avaliar os ambientes represados, serão consideradas as usinas que possuam reservatórios, no caso as PCHs e UHEs. A gestão dos reservatórios de hidrelétricas é imprescindível para a proteção do potencial hidráulico e do aproveitamento hidrelétrico. Existem diversos problemas relacionados aos ambientes aquáticos represados, como assoreamento; proliferação de macrófitas; bloom de cianobactérias e presença de espécies invasoras, como o mexilhão dourado (Limnoperna fortunei). Recentemente, devido à escassez de chuva nos últimos anos enfrentada em várias regiões do Brasil, uma das questões mais graves é a diminuição do volume de água nos reservatórios.

Os principais fatores associados às modificações passadas por um reservatório em relação à qualidade de suas águas (Tundisi, 2003) são: 
a) volume de água;

b) morfometria da represa;

c) características da área de inundação (geomorfologia da região, tipo de solo,uso do solo, cobertura vegetal);

d) usos da bacia hidrográfica na área de inundação;

e) qualidade inicial da água dos rios formadores da represa.

Grande parte dos estudos sobre ambientes aquáticos lênticos (de água parada ou de pouco fluxo) são sobre lagos. Embora eles se diferenciem dos reservatórios, desde sua origem (natural, no caso dos lagos) até em suas características (morfometria, renovação das águas) esses estudos também foram usados como referência deste trabalho.

As classificações de corpos de água (ambientes lóticos e lênticos) surgiram na Europa por meio da DQA - Diretiva Quadro das Águas (2000), documento oficial da Comunidade Europeia, criado com o objetivo de estabelecer um marco estratégico para a gestão dos ecossistemas aquáticos e estabelecer um quadro de ação comunitária no domínio da política da água por meio da coordenação, integração e adaptação das estruturas normativas e institucionais dos Estados Membros aos princípios gerais de proteção.

A essa classificação dos ambientes aquáticos se dá o nome de tipificação. O quadro 01 contempla as variáveis utilizadas na tipificação de reservatórios e lagos baseado em experiências internacionais.

Quadro 01: Exemplos internacionais de tipificação de reservatórios e lagos.

\begin{tabular}{|c|c|}
\hline Local, documento & Variáveis \\
\hline $\begin{array}{l}\text { União Europeia (Diretiva } \\
\text { Quadro das Águas) }\end{array}$ & $\begin{array}{l}\text { Altitude área de drenagem, profundidade, geologia, latitude, longitude, } \\
\text { profundidade média,forma do lago, tempo de residência, temperatura } \\
\text { ar,amplitude térmica do ar,capacidade de neutralizar ácidos,estado de } \\
\text { referencia de concentração de nutrientes, composição media do substrato, } \\
\text { flutuação do nível das aguas, características de mistura - estratificação } \\
\text { (DQA, 2000) }\end{array}$ \\
\hline $\begin{array}{c}\text { Portugal (Qualidade } \\
\text { Ecológica e Gestão } \\
\text { Integrada de Albufeiras) }\end{array}$ & $\begin{array}{l}\text { Área inundada, altitude, profundidade média, altitude latitude, longitude, } \\
\text { geologia,profundidade máxima, volume, desenvolvimento da margem, índice } \\
\text { de forma, temperatura média do ar, variação média do nível da água, } \\
\text { profundidade média, grau de mineralização,dureza da água,área da bacia de } \\
\text { drenagem, declive médio da bacia de drenagem, densidade da rede de } \\
\text { drenagem, distância à nascente, distância à foz, número de afluentes, }\end{array}$ \\
\hline & $\begin{array}{l}\text { número de ordem máximo, evapotranspiração real média anual, precipitação } \\
\text { média anual na bacia etemperatura média anual na bacia. (INAG, 2009). }\end{array}$ \\
\hline $\begin{array}{l}\text { Chile (Classificação de } \\
\text { corpos de água) }\end{array}$ & $\begin{array}{l}\text { Altitude, tamanho do lago, condutividade elétrica, profundidade, estratificação. } \\
\text { (Fuente et al, 2010). }\end{array}$ \\
\hline
\end{tabular}

Cadernos do Leste 
Coreia (Caracterização os efeitos do uso do solo e morfometria na qualidade da água de reservatórios)
Uso do solo; altitude, altura de margem; perímetro, comprimento e área do reservatório; área da bacia de contribuição; oxigênio dissolvido; demanda química de oxigênio; sólidos suspensos totais; nitrogênio total; fósforo total; clorofila a. (Park et al, 2014).

De acordo com o Serviço Geológico dos Estados Unidos (USGS, 2015) os fatores básicos que melhor definem um lago são clima, inputsatmosféricos (precipitação, partículas), rocha e solo da bacia hidrográfica, uso do solo e morfometria. Os maiores problemas ambientais nos lagos são: proliferação excessiva de algas, aporte de sedimentos, depleção de oxigênio, grandes variações do nível de água e espécies exóticas.

A morfologia de um ambiente aquático exerce uma marcante influência em todas as suas características limnológicas, condicionando fortemente a interação dos componentes físicos, químicos e biológicos do sistema (Sperling, 1999).

\section{2- METODOLOGIA}

O presente trabalho faz parte da dissertação de Mestrado do Programa de Pós-Graduação em Análise e Modelagem de Sistemas Ambientais do Instituto de Geociências da Universidade Federal de Minas Gerais, integrando o projeto "Utilização de Índice de Integridade Ecológica para Classificar a Qualidade de Ambientes Aquáticos de Minas Gerais" Fapemig e Cemig, realizado no Instituto Senai de Tecnologia em Meio Ambiente.

Inicialmente, em uma abordagem ampla, utilizou-se o conceito de Ecorregiões Aquáticas para divisão do Estado. Estas são áreas em que condições ambientais ou conjuntos de espécies são possivelmente homogêneos quando comparadas à heterogeneidade observada em áreas mais amplas (MMA, 2009). O limite das ecorregiões aquáticas em MG foi baseado na divisão estabelecida por Abellet al (2008) e na Resolução CNRH No 32 de 2003 (Regiões hidrográficas brasileiras).

Os reservatórios estudados foram selecionados da Cemig (Companhia Energética de Minas Gerais), totalizando em dezenove (figura 01). Para a elaboração dos estudos, foram levantadas cerca de 60 variáveis. Estas informações foram obtidas de fontes variadas, a saber: Cemig, IBGE (Instituto Brasileiro de Geografia e Estatística), CPRM (Serviço Geológico do Brasil), Siságua (Sistema de informação de qualidade da água dos reservatórios da Cemig), SRTM (Missão Topográfica Radar Shuttle), WorldClim (base de dados climáticos globais), além de dados medidos e calculados através da base cartográfica do projeto. Em seguida esse volume de variáveis foi reduzido para 25 , levando em consideração a qualidade e existência dos dados para todos os reservatórios. 
Figura 01: Localização dos reservatórios selecionados para o estudo em Minas Gerais.

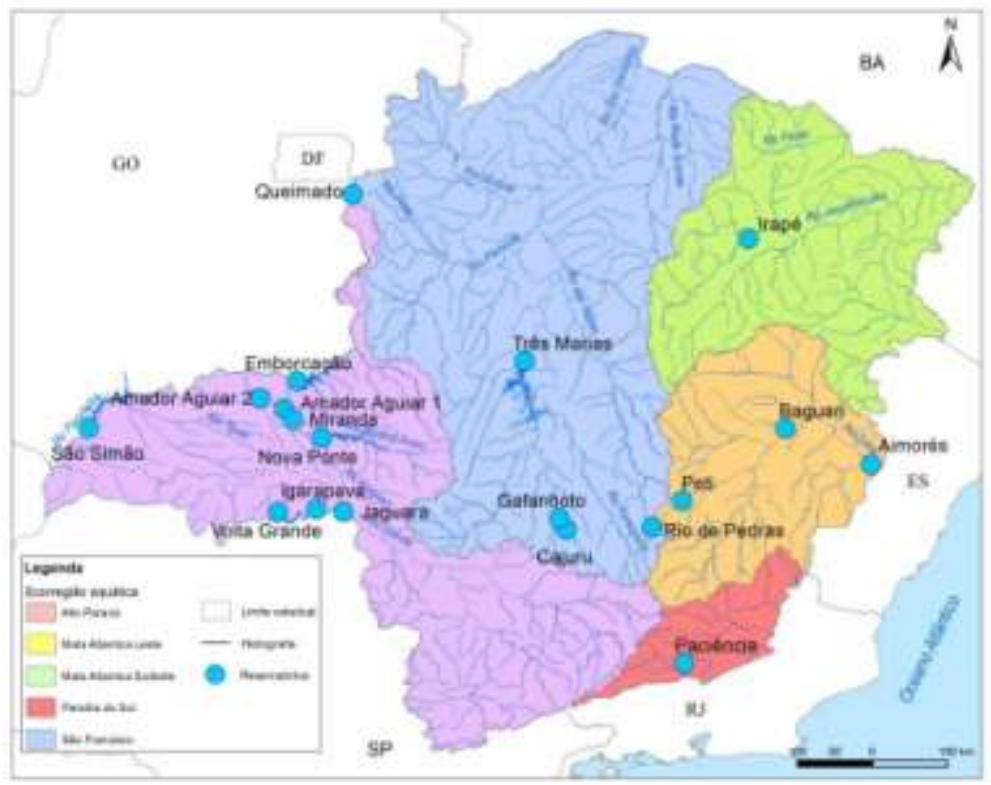

Fonte: elaborado pela autora.

Visando selecionar as variáveis mais representativas e eliminar sobreposições, foi aplicada a Análise de Componentes Principais (ACP) no softwareStatistica ${ }^{\mathrm{TM}}$ 7. De acordo com Carlos Alberto Varella (2008), está é uma:

técnica estatística multivariada que consiste em transformar um conjunto de variáveis originais em outro conjunto de variáveis de mesma dimensão denominadas de componentes principais. Os componentes principais apresentam propriedades importantes: cada componente principal é uma combinação linear de todas as variáveis originais, são independentes entre si e estimados com o propósito de reter, em ordem de estimação, o máximo de informação, em termos da variação total contida nos dados. A análise de componentes principais é associada à ideia de redução de massa de dados, com menor perda possível da informação.

Após a seleção das variáveis pela ACP foi utilizada a análise de agrupamento (cluster), por meio de distância Euclidiana, no softwareStatistica ${ }^{\text {TM }}$ 7. A análise de cluster tem por objetivo "agrupar elementos de dados baseando-se na similaridade entre eles. Os grupos são determinados de forma a obter-se homogeneidade dentro dos grupos e heterogeneidade entre eles" (DONI, 2004).

Foram utilizados dois métodos de agrupamento: Joining (treeclustering) e Kmeansclustering. O primeiro reúne os elementos em árvore, mostrando em um dendograma os grupos formados e a distância de ligação entre eles. Os objetos são agrupados em grupos cada vez maiores, segundo o aumento da dissimilaridade (distância) entre eles. A ordem em que os elementos estão dispostos no dendograma é aquela em que cada objeto entrou no agrupamento. Cadernos do Leste 
Para esta etapa foi utilizada a Distância Eucidiana, que é uma medida de dissimilaridade, que mede a distância entre dois pontos, pela soma da raiz quadrada da diferença entre x e y em suas respectivas dimensões $\left(\mathrm{d}_{\mathrm{ab}}=\left[\sum_{j=1}^{p}(\mathrm{xaj}-\mathrm{xbj}) 2\right] 1 / 2\right)$. A regra de aglomeração ou ligação utilizada para calcular a árvore de matriz de distância foi a "Unweightedpair-groupaverage", em que a distância entre dois clusters é calculada como a distância média entre todos os pares de objetos nos dois diferentes clusters" (Câmara, 2009).

No outro métodos usado, o K-meansclustering, a distância entre dois grupos é calculada como a distância média entre todos os pares de objetos em dois grupos diferentes (Statistica ${ }^{\mathrm{TM}}$ 7, manual eletrônico). Essa etapa foi realizada como uma forma de validar o método anterior. $\mathrm{O}$ método permite avaliar a distância de cada elemento, no caso cada reservatório, do centro de seu grupo, mostrando qual indivíduo mais se afasta do seu cluster

Por meio do agrupamento dos reservatórios, foram analisadas as características mais relevantes que conferiram a homogeneidade aos grupos formados. O reservatório de Paciência não foi avaliado nas análises devido à ausência de grande parte dos dados.

\section{3- RESULTADO}

$\mathrm{Na} \mathrm{ACP}$, os fatores gerados mostram que as duas primeiras componentes explicam cerca de $55 \%$ do modelo. A primeira componente explica $36,57 \%$ e a segunda $18,29 \%$ da variância (figura 02). As variáveis que são mais significativas para a representam são as que se aproximam da circunferência unitária.

Analisando a influência de cada variável nos dois primeiros fatores (tabela 01), nota-se que na primeira componente a variável que mais se correlaciona é de comprimento $(0,906)$, seguida do volume total $(-0,898)$ e perímetro $(-0,871)$. Já a de menor correlação é a de altitude $(0,028)$. $\mathrm{Na}$ segunda componente, diferente do que o ocorre na primeira, a altitude é a de maior correlação ($0,828)$, seguida da temperatura média anual $(0,827)$. A de menor influência é o volume total ($0,07)$. 
Figura 02: Análise de componentes principais.

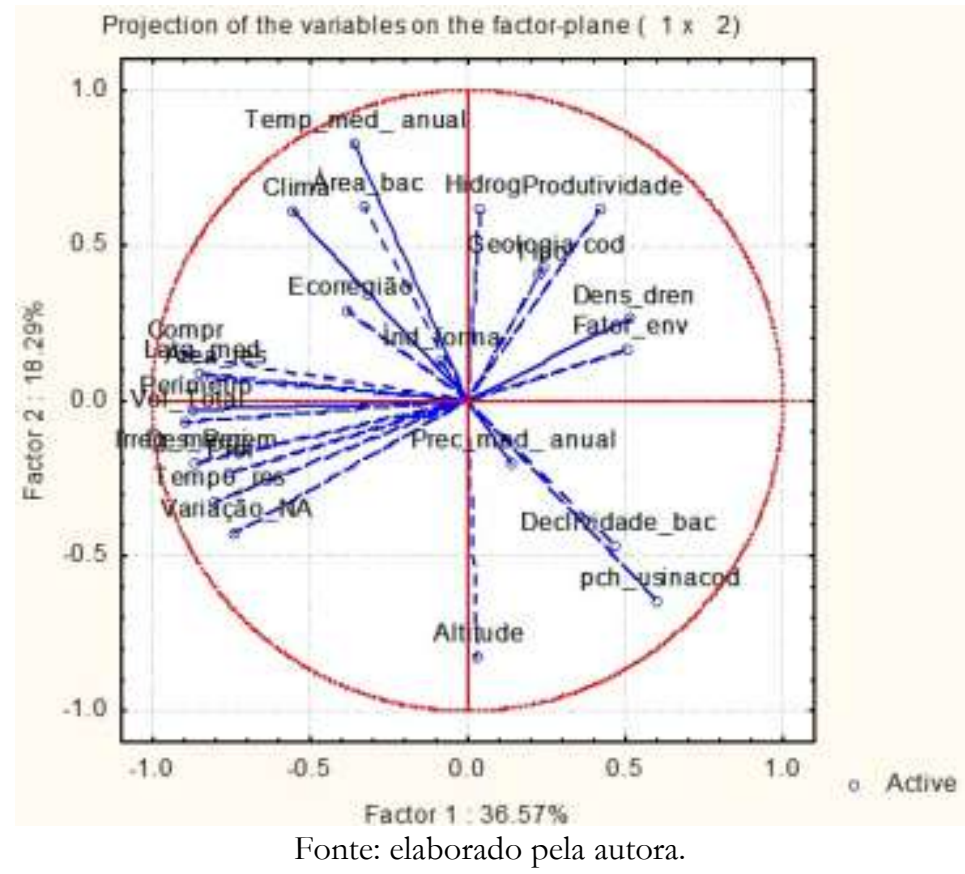

Com o objetivo de selecionar as variáveis mais significativas e eliminar sobreposições, foram escolhidas as variáveis com fator acima de 0,5 e eliminadas aquelas que tinham a mesma resposta no modelo. Quanto às variáveis altamente correlacionadas, tem-se o perímetro, desenvolvimento de perímetro e irregularidade das margens. Esse resultado é esperado, uma vez que o desenvolvimento de perímetro $\left(\right.$ perímetro $/ 2 \pi r^{2}$ e irregularidade das margens $($ perimetro $/ \sqrt{\sqrt{\text { area. } \pi}})$ derivam de fórmulas que dependem do valor do perímetro. Decidiuse por escolher o desenvolvimento de perímetro, uma vez que é um dos parâmetros mais utilizados em limnologia para descrever a morfometria dos corpos de água. (Wetzel apud Assireuet al, 2009). 
Tabela 01:Variáveis e fatores da Análise de Componentes Principais.

\begin{tabular}{|c|c|c|}
\hline VARIAVEL $\quad$ FATOR & Fator 1 & Fator 2 \\
\hline Ecorregião & $-0,379677$ & 0,292753 \\
\hline Tipo & 0,225155 & 0,402848 \\
\hline PCH ou UHE* & 0,603258 & $-0,646884$ \\
\hline Altitude* & 0,027924 & $-0,827611$ \\
\hline Geologia & 0,239053 & 0,426641 \\
\hline Comprimento* & $-0,906066$ & 0,152707 \\
\hline Largura média* & $-0,850868$ & 0,085459 \\
\hline \multirow[t]{2}{*}{ Indice de forma } & $-0,088549$ & 0,124178 \\
\hline & & \\
\hline Variação do nível* & $-0,741391$ & $-0,425368$ \\
\hline Área da bacia* & $-0,325845$ & 0,623339 \\
\hline Declividade da bacia & 0,468019 & $-0,466256$ \\
\hline Densidade de drenagem* & 0,517255 & 0,262548 \\
\hline Precipitação média anual & 0,137912 & $-0,205039$ \\
\hline Temperatura média anual* & $-0,362765$ & 0,826570 \\
\hline Área do reservatório* & $-0,811572$ & 0,069944 \\
\hline Fator de envolvimento* & 0,506437 & 0,165851 \\
\hline Volume total* & $-0,897834$ & $-0,070467$ \\
\hline Profundidade* & $-0,759872$ & $-0,234974$ \\
\hline Perímetro & $-0,870567$ & $-0,029308$ \\
\hline $\begin{array}{l}\text { Desenvolvimento de } \\
\text { perímetro* }\end{array}$ & $-0,867616$ & $-0,203154$ \\
\hline Irregularidade das margens & $-0,867616$ & $-0,203154$ \\
\hline Produtividade & 0,418257 & 0,613652 \\
\hline Tempo de residência* & $-0,799941$ & $-0,325304$ \\
\hline Hidrogeologia* & 0,039114 & 0,617734 \\
\hline
\end{tabular}

Fonte: elaborado pela autora.

A seguir, no quadro 02, são descritas as dezesseis variáveis selecionadas para classificar os reservatórios. 
Quadro 02: Variáveis selecionadas e descrição.

\begin{tabular}{|c|c|}
\hline Variável & Descrição \\
\hline PCH ou UHE & $\begin{array}{l}\text { Classificação das usinas em pequenas centrais hidrelétricas ou usinas hidrelétricas de } \\
\text { energia. Fonte do dado: Cemig }\end{array}$ \\
\hline Altitude & Altitude do barramento em relação ao nível do mar, obtida pelo Google Earth. \\
\hline Comprimento & $\begin{array}{l}\text { Extensão do espelho de água, partindo do início até a barragem, considerando o } \\
\text { curso de água principal formador do reservatório. Medido no ArcGIS. }\end{array}$ \\
\hline Largura média & Valor médio de larguras medidas no reservatório, medidas no ArcGIS. \\
\hline Variação do nível & Flutuação do nível das águas. Fonte: Cemig. \\
\hline Área da bacia & $\begin{array}{l}\text { Área drenada pelo sistema pluvial inclusa entre seus divisores topográficos, projetada } \\
\text { em plano horizontal, medida no ArcGIS. }\end{array}$ \\
\hline Densidade de drenagem & $\begin{array}{l}\text { Calculado pela relação entre o comprimento total dos canais ou rios com a área da } \\
\text { bacia hidrográfica. }\end{array}$ \\
\hline $\begin{array}{l}\text { Temperatura média } \\
\text { anual }\end{array}$ & $\begin{array}{l}\text { Média anual de temperatura na área do reservatório entre os anos de } 1950 \text { e } 2000 . \\
\text { Obtidos do WorldClim. }\end{array}$ \\
\hline Área do reservatório & Área do espelho de água, medida no software ArcGIS. \\
\hline Fator de envolvimento & $\begin{array}{l}\text { Calculado pela razão entre a área da bacia hidrográfica do reservatório e a área do } \\
\text { reservatório, sendo adimensional. Para lagos naturais os valores mais comuns são da } \\
\text { ordem de algumas dezenas, e para reservatórios artificiais, esse valor pode atingir } \\
\text { centenas, uma vez que as bacias hidrográficas são geralmente bem maiores em } \\
\text { relação ao tamanho do corpo de água (SPERLING, 1999). }\end{array}$ \\
\hline Volume total & Somatório do volume morto com o volume útil. Fonte: Cemig \\
\hline Profundidade & Razão entre o volume máximo e a área máxima do reservatório. Fonte: Cemig \\
\hline $\begin{array}{l}\text { Desenvolvimento de } \\
\text { perímetro }\end{array}$ & $\begin{array}{l}\text { Relação entre o perímetro do lago e a circunferência de um círculo que possua a } \\
\text { mesma área do lago, sendo adimensional. Quanto mais próximo de uma forma } \\
\text { circular for o reservatório, mais o valor de Dp se aproxima da unidade. }\end{array}$ \\
\hline Tempo de residência & $\begin{array}{l}\text { Tempo gasto para o reservatório renovar suas águas. Quanto menor é esse tempo, } \\
\text { melhor, uma vez que os poluentes são expulsos antes que causem danos maiores à } \\
\text { biota aquática. (UFRRJ, 2015). Fonte do dado: Cemig }\end{array}$ \\
\hline Hidrogeologia & $\begin{array}{l}\text { Domínios hidrogeológicos. Classifica se o aquífero é poroso (alta permeabilidade), } \\
\text { fissural ou misto. Fonte: CPRM, } 2006 .\end{array}$ \\
\hline Clima & Baseado na classificação de Nimerapud IBGE (2002). \\
\hline
\end{tabular}

O passo seguinte foi o método estatístico de Análise de Cluster. Esta análise agrupou os reservatórios mais semelhantes, por meio do método "Joining (treeclustering)" do Statistica TM 8. Formou-se dois grandes grupos na distância de ligação 6, separando as UHEs de PCHs. Na distância de aproximadamente 3,5; os reservatórios UHEs foram divididos em dois grupos (Figura 03).

Figura 03: Dendograma com agrupamento de reservatórios. 


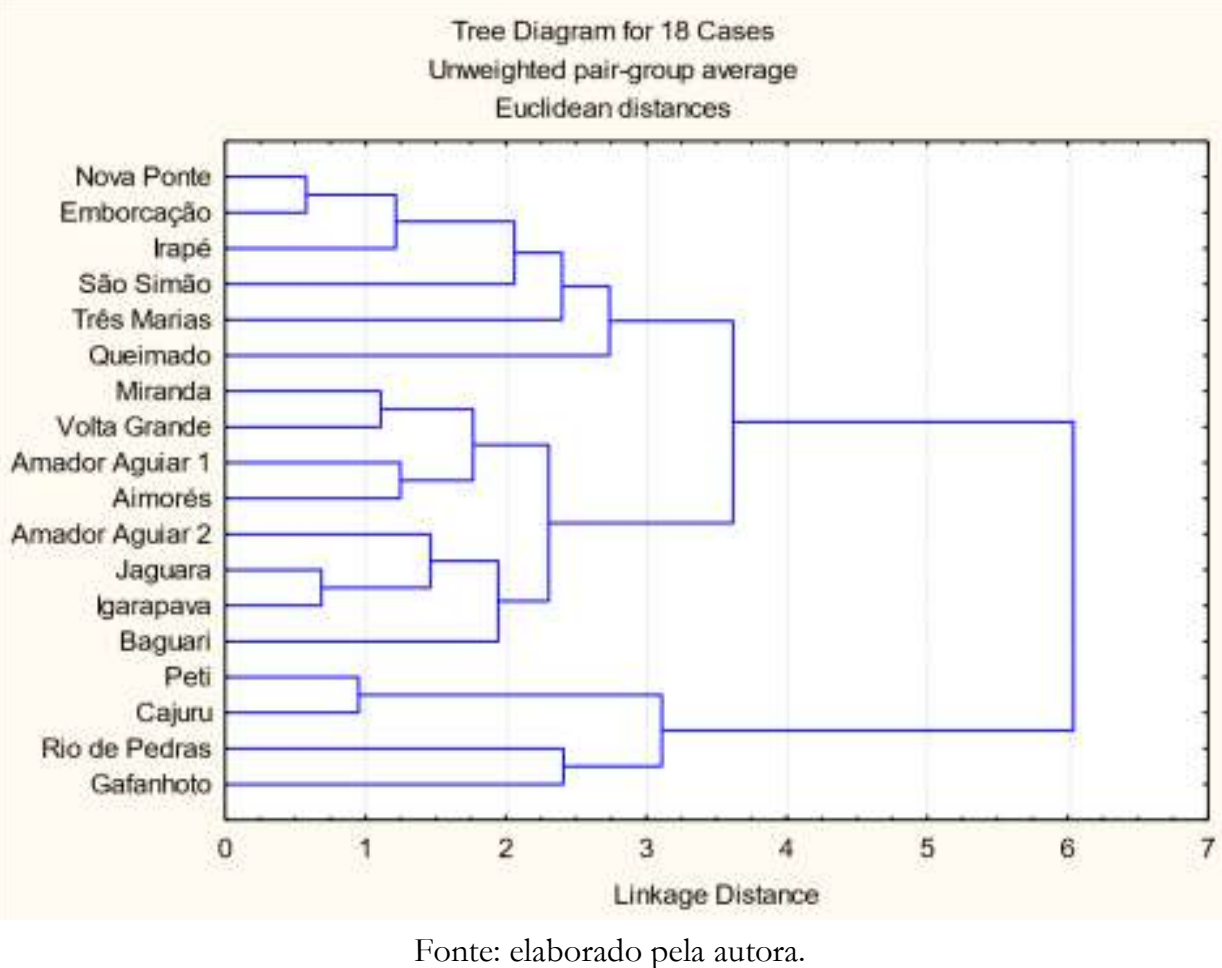

Avaliando as informações contidas nas variáveis, as características mais relevantes dos três grupos formados foram:

- Grupo I: Nova Ponte, Emborcação, Irapé, São Simão, Três Marias e Queimado. UHEs, com maiores dimensões, como comprimento; largura; áreas do reservatório e da bacia de contribuição; volume e profundidade.

- Grupo II: Miranda, Volta Grande, Amador Aguiar 1, Aimorés, Amador Aguiar 2, Jaguara, Igarapava e Baguari. UHEs, com dimensões intermediárias, possuem os menores tempos de residência.

- Grupo III: Peti, Cajuru, Rio de Pedras e Gafanhoto. PCHs, reservatórios menores e menos profundos, com temperaturas médias mais baixas e localizados apenas em aquíferos fissurais.

Utilizando o método "K-meansClustering", foi definido o número de três clusters com base na etapa anterior. Assim como no método treeclustering, cada grupo formado continha os mesmos reservatórios. A tabela 02 mostra as distâncias de cada reservatório do centro do seu respectivo cluster.

Tabela 02: distância de cada reservatório do centro do seu grupo (cluster).

\begin{tabular}{|c|c|c|c|c|c|}
\hline $\begin{array}{c}\text { Reservatório } \\
\text { (Grupo I) }\end{array}$ & Distância & $\begin{array}{c}\text { Reservatório } \\
\text { (Grupo II) }\end{array}$ & Distância & $\begin{array}{c}\text { Reservatório } \\
\text { (Grupo III) }\end{array}$ & Distância \\
\hline Emborcação & 0,211331 & Amador Aguiar 2 & 0,196900 & Peti & 0,299701 \\
\hline Irapé & 0,235113 & Miranda & 0,349689 & Cajuru & 0,436348 \\
\hline
\end{tabular}

Cadernos do Leste

Artigos Cientificos

Belo Horizonte, Jan.-Dez. Vol.16, n¹6, 2016 


\begin{tabular}{|c|c|c|c|c|c|}
\hline Nova Ponte & 0,236664 & Aimorés & 0,325347 & Gafanhoto & 0,458520 \\
\hline São Simão & 0,423943 & Amador Aguiar 1 & 0,269997 & Rio de Pedras & $\mathbf{0 , 4 7 2 4 7 3}$ \\
\hline Três Marias & 0,456966 & Igarapava & 0,300294 & - & - \\
\hline Queimado & $\mathbf{0 , 5 1 4 3 4 6}$ & Jaguara & 0,313298 & - & - \\
\hline- & - & Volta Grande & 0,414420 & - & - \\
\hline- & - & Baguari & $\mathbf{0 , 5 0 8 7 4 8}$ & - & - \\
\hline
\end{tabular}

Fonte: elaborado pela autora.

Os reservatórios em negrito são aqueles que mais se afastaram do seu grupo. De acordo com o valor de cada variável, o distanciamento destes pode ser devido às seguintes características:

- Queimado -maior altitude e menores: comprimento, área da bacia e do reservatório, densidade de drenagem, volume total, profundidade e perímetro dentro do grupo I.

- Baguari - maior fator de envolvimento e menores:comprimento, área do reservatório, volume total, profundidade, perímetro e tempo de residência dentro do grupo II

- Rio de Pedras: maior altitude e menores variação de nível, volume total, profundidade; único do grupo no clima mesotérmico brando 4 a 5 meses secos, dentro do grupo III.

A tabela 03 contém a média dos valores dos atributos das variáveis, separados nos três grupos citados acima e a figura 04 mostra a localização dos reservatórios por grupos.

Tabela 03: Médias dos valores das variáveis selecionadas por grupo. 


\begin{tabular}{|c|c|c|c|}
\hline VARIÁVEL GRUPO & $I(6)$ & II (8) & III (4) \\
\hline $\mathrm{PCH} / \mathrm{UHE}$ & UHE & UHE & $\mathrm{PCH}$ \\
\hline Altitude & 608,1666667 & 456,875 & 767 \\
\hline Comprimento & 105138,3333 & 46996,25 & 9675 \\
\hline Largura média & 2344,5 & 990 & 392,7 \\
\hline Variação nível & 25,63333333 & 1,2125 & 6,5075 \\
\hline Área da bacia & 47711,1 & 43257,84 & 1535,843 \\
\hline Densidade de drenagem & 0,489689705 & 0,620879 & 0,675006 \\
\hline Temp. média anual & 22,93333333 & 23,3875 & 20,5 \\
\hline Área reservatório & 435,7633333 & 49,28375 & 6,1925 \\
\hline Fator de envolvimento & 115,6414642 & 1634,54 & 2780,716 \\
\hline Volume total & 11504,47 & 676,0463 & 72,4025 \\
\hline Profundidade média & 26,82020376 & 12,19078 & 8,012971 \\
\hline Perímetro & 1151,576667 & 152,7238 & 42,075 \\
\hline Tempo de residência & 225,6685 & 1,300125 & 19,19475 \\
\hline Hidrogeologia & $\begin{array}{l}\text { Fissural (4), misto (1) e } \\
\text { poroso (1) }\end{array}$ & $\begin{array}{l}\text { Fissural (4), misto (1) e } \\
\text { poroso (3) }\end{array}$ & Fissural (4) \\
\hline Clima & $\begin{array}{l}\text { Quente } 4 \text { a } 5 \text { meses secos (5) e } \\
\text { quente } 1 \text { a } 3 \text { meses secos (1) }\end{array}$ & $\begin{array}{l}\text { Quente } 4 \text { a } 5 \text { meses } \\
\qquad \sec \cos (8)\end{array}$ & $\begin{array}{c}\text { Subquente } 4 \text { a } 5 \text { meses secos (3) } \\
\text { e mesotérmico brando } 4 \text { a } 5 \\
\text { meses secos (1) }\end{array}$ \\
\hline
\end{tabular}

Fonte: elaborado pela autora. 
Figura 04: localização dos reservatórios em Minas Gerais separados por grupos.

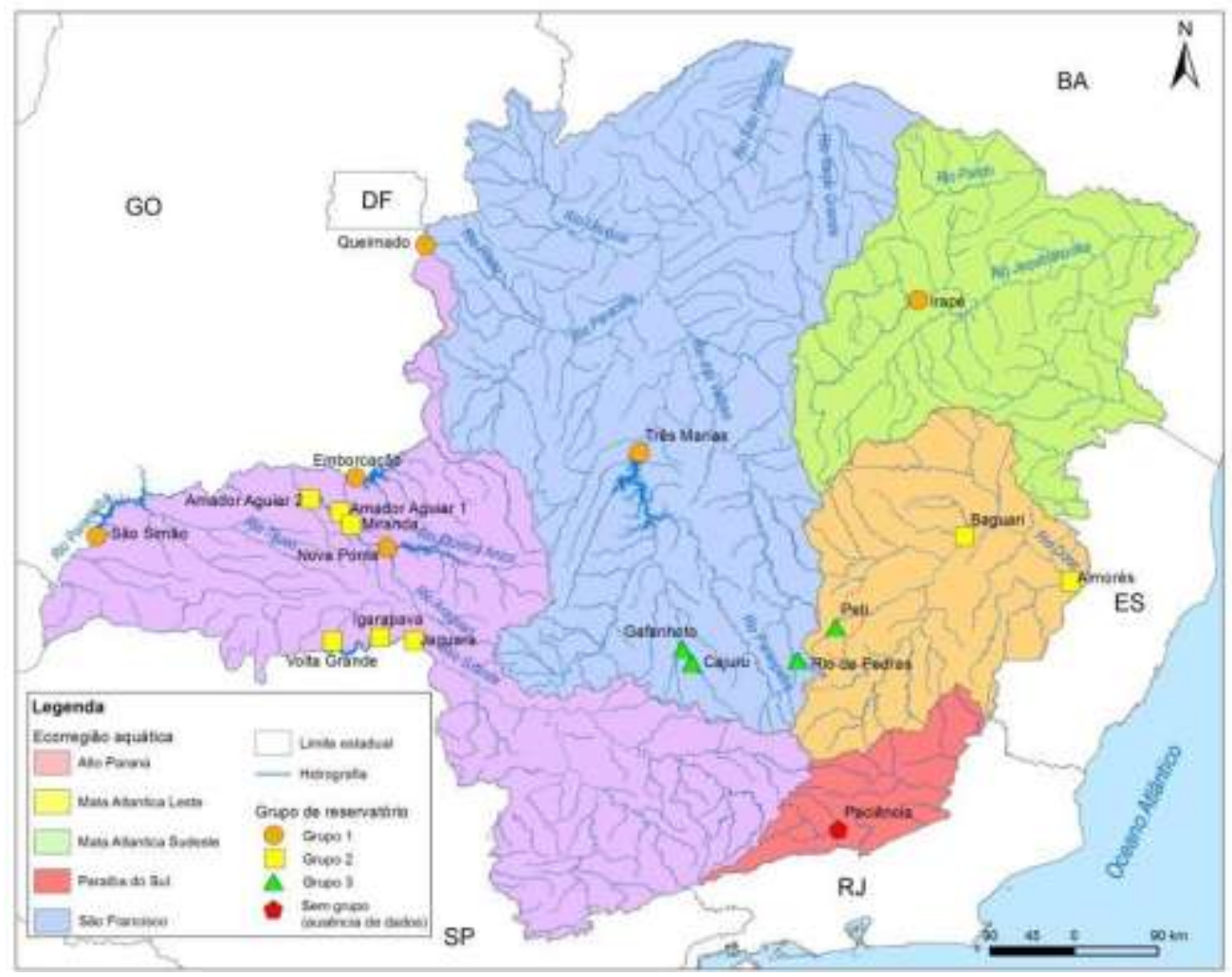

Fonte: elaborado pela autora.

\section{4- DISCUSSÃO}

Os resultados das análises mostraram que, em linhas gerais, a dimensão do reservatório foi determinante para a formação dos grupos. Para aperfeiçoamento do método, seria pertinente a incorporação de outras variáveis além das morfométricas, como as físicoquímicas. Para tanto, fazse necessário avaliar a disponibilidade e qualidade dos dados requeridos. Para resultados ainda mais robustos, é válido o estudo de outros reservatórios de usinas hidrelétricas além dos selecionados pela Cemig.

A próxima etapa do projeto "Utilização de Índice de Integridade Ecológica para Classificar a Qualidade de Ambientes Aquáticos de Minas Gerais" será a validação da tipificação por meio de resultados biológicos de campanhas de coleta. Essa validação tem por objetivo verificar se o método utilizado foi eficiente baseado nas comunidades encontradas nos reservatórios de áreas de estudo, e apontará se de fato os resultados obtidos com a tipificação aqui apresentada terão repostas biológicas homogêneas para os grupos formados. 


\section{5- CONCLUSÃO}

O Brasil, devido a sua dependência de energia hidrelétrica, necessita de gestão e governança eficientes no que tange esse setor. A organização e sistematização dos dados se tornam subsídio para órgãos, empresas e governos realizarem esse gerenciamento. Os reservatórios são ambientes que desde sua origem acarretam grandes mudanças no ambiente e ao longo de sua vida útil estão sujeitos a uma série de problemas, ressaltando a importância de estudá-los.

Por meio dos processos estatísticos utilizados, formou-se três grupos de reservatórios, do qual a dimensão foi o principal definidor de suas características. Considerando a média das variáveis, o primeiro grupo foi dos maiores reservatórios de UHEs, com grandes áreas, perímetros e profundidade. O segundo foi dos reservatórios de UHEs com tamanho intermediário dentro da amostra, e com os menores tempos de detenção. O terceiro grupo contempla as PCHs, com menores perímetros, área e menor profundidade. A etapa seguinte desse trabalho será a validação do modelo por meio de resultados biológicos, que irão confirmar ou não se os tipos de reservatórios possuem homogeneidade em suas características.

O presente trabalho contribui para os estudos de reservatórios em Minas Gerais, uma vez que se trata de uma metodologia inédita no país, baseada nas experiências de internacionais para a tipificação desses ambientes.

\section{AGRADECIMENTOS}

À equipe do Instituto SENAI de Tecnologia em Meio Ambiente participante do projeto "Utilização de Índice de Integridade Ecológica para Classificar a Qualidade de Ambientes Aquáticos de Minas Gerais", pela colaboração, estrutura e pelo fornecimento de dados.

À equipe da Cemig Geração e Transmissão pela autorização do uso dos dados para realização de dissertação de mestrado.

À Fundação de Amparo à Pesquisa do Estado de Minas Gerais (FAPEMIG), a Cemig Geração e Transmissão (Cemig GT) e Agência Nacional de Energia Elétrica - ANEEL (P \& D), pelo financiamento do projeto "Utilização de Índice de Integridade Ecológica para Classificar a Qualidade de Ambientes Aquáticos de Minas Gerais”.

\section{REFERÊNCIAS BIBLIOGRÁFICAS}

ABELL et al. Freswaterecoregions of the world. A new map of biogeographic.Units for FreshwaterbiodiversityConservation. BioScience.58: p. 403-414, 2008. 
ANEEL - Agência Nacional de Energia Elétrica. Matriz de energia elétrica. Disponível em $<$ http://www.aneel.gov.br/aplicacoes/capacidadebrasil/OperacaoCapacidadeBrasil.cfm>, acesso em setembro de 2015.

ASSIREU, ArcilanTrevenzoli; FREITAS, Ramon Morais de; NOVO, Evlyn Márcia Leão de Moraes; PACHECO, Felipe Rolan da Silva; STECH, José Luiz. Índice de criticalidade da qualidade da água em lagos e reservatórios: Uma proposta de metodologia baseada em variáveis estáticas e dinâmicas. Anais XIV Simpósio de Sensoriamento Remoto, 2009. Disponível em <http://marte.sid.inpe.br/col/dpi.inpe.br/sbsr@80/2008/11.16.16.23/doc/46394646.pdf>, acesso em novembro de 2015.

CÂMARA, Fernando Portela. Psiquiatria e estatística - Parte II: fundamentos da análise de clusters (classificação numérica). 2009. Disponível em < http://www.polbr.med.br/ano09 /cpc0109.php>, acesso em novembro de 2015.

CAMPAGNOLI, Fernando e DINIZ, Noris Costa. Gestão de Reservatórios de Hidrelétricas. São Paulo, Oficina dos textos, 2012.

CPRM - Serviço Geológico do Brasil. Mapa de domínios hidrogeológicos. 2006.

Disponível em

$<$ http://www.cprm.gov.br/publique/cgi/cgilua.exe/sys/start.htm?infoid=756\&sid=9>, acesso em abril de 2014.

DONI, Marcelo Viana. Análise de cluster: métodos hierárquicos e de particionamento. São Paulo, 2004. Disponível em: <http://meusite.mackenzie.com.br/rogerio/tgi/2004Clus ter.PDF>, acesso em setembro de 2015.

DQA, Directiva Quadro da Agua. Directiva 2000/60/CEdo Parlamento Europeu e do Conselho de 23 de outubro de 2000. Jornal Oficial dasComunidades Europeias. L 327, 22.12.2000, 72pp.

ENERGISA. Usinas hidrelétricas. Disponível em <http://geracao.grupoenergisa.com. br/paginas/nossos-negocios/pchs.aspx>, acesso em agosto de 2015.

FUENTE, Andrés de la; SABANDO, MaríaCatalina; PÉREZ, Jorge. Clasificación de cuerpos de agua: informe final. Santiago: Universidad de Chile, 2010. Disponível em:

<http:..www.sinia.cl.1292.articles-48828_recurso_1.pdf>. Acesso em junho de 2014.

IBGE - Instituto Brasileiro de Geografia Estatística. Mapa de clima. Disponível em $<$ http://atlasescolar.ibge.gov.br/images/atlas/mapas_brasil/brasil_clima.pdf $>$, acesso em março de 2014.

INAG I. P. Instituto da Água I.P. 2009. Qualidade ecológica e gestão integrada de albufeiras. Disponível em: <http://www.apambiente.pt/dqa/assets/qualidadeecol\%CC3\%B3gica-egest $\%$ C3\%A3o-integrada-de-albufeiras.pdf $>$, acesso em março de 2015. 
MMA - Ministério do Meio Ambiente. Ecorregiões Aquáticas. Disponível em <http://www.mma.gov.br/estruturas/sbf_chm_rbbio/_arquivos/eco_srhu_mma_72.pdf>, acesso em outubro de 2015.

PARK, Young-Seuk; KWON, Yong-Su; HWANG, Soon-Jin e PARK, Sangkyu.Characterizingeffects of landscape and morphometricfactorsonwaterquality of reservoirsusing a self-organizingmap.Elsevier, 2014. Disponível em < http://www.sciencedirect.com/science/article/pii/S1364815214000413>, acesso em agosto de 2015.

RESOLUCAO CNRH No. 32, de 15 de outubro de 2003. Publicada no DOU em 17.12.2003.

SISÁGUA - Sistema de informação de qualidade da água dos reservatórios da Cemig. Disponível em < http://www2.cemig.com.br/sag/>, acesso em fevereiro de 2015.

SPERLING, Eduardo von. Morfologia de lagos e represas. Belo Horizonte, DESA Universidade Federal de Minas Gerais, 1999.

SRTM - Shuttle Radar Topography Mission.Download. Disponível em <http://www.relevobr.cnpm.embrapa.br/download/>, acesso em janeiro de 2014.

STATISTICA TM 7 - Manual Eletrônico. StatSoft.

TUNDISI, José Galizia. Reservatórios como sistemas complexos. In: HENRY, Raoul. Ecologia de reservatórios. São Paulo, FapespFundibio, 1999. 21-27.

UFRRJ - Universidade Federal Rural do Rio de Janeiro. Parâmetros hidrológicos. Disponível em $<$ http://www.ufrrj.br/institutos/it/de/acidentes/hid.htm>, acesso em agosto de 2015.

USGS - Serviço Geológico dos Estados Unidos. Lagos e reservatórios. Disponível em <http://water.usgs.gov/edu/earthlakes.html>, acesso em novembro de 2015.

VARELLA, Carlos Alberto Alves. Análise de componentes principais. Universidade Federal Rural do Rio de Janeiro, 2008. Disponível em

$<$ http://www.ufrrj.br/institutos/it/deng/varella/Downloads/multivariada\%20aplicada $\% 20$ as $\% 2$ 0ciencias $\% 20$ agrarias/Aulas/analise $\% 20 \mathrm{de} \% 20$ componentes $\% 20$ principais.pdf $>$, acesso em outubro de 2015.

WordClim - Global Climate Data. Download de dados de temperatura e precipitação. Disponível em < http://www.worldclim.org/download>, acesso em abril de 2015. 